AR 3043

Klaus Ceynowa

\title{
Vom Wert des Sammelns und vom Mehrwert des Digitalen - Verstreute Bemerkungen zur gegenwärtigen Lage der Bibliothek
}

Zusammenfassung: Es ist Zeit für die Renaissance der Bibliothek als Sammlung, gerade im digitalen Zeitalter. Die vielfältigen Nutzungsszenarien digitaler Inhalte verlangen ganz traditionell ihren Besitz und ihre Dauerverfügbarkeit. Im Sammlungsgedanken sind sich gedruckter, digitaler und multimedialer Content näher als die gegenwärtigen Dichotomien des Virtuellen und Realen glauben machen wollen. Wer heute von "Sammlungen" spricht, darf von "Fachinformationsdiensten" als Arkanum eines ganz dem gegenwärtigen Bedarf verpflichteten Bibliotheksverständnisses nicht schweigen. Es entpuppt sich beim näheren Zusehen als Entlastungsstrategie von den mit einer Sammlung verbundenen Ansprüchen und Zumutungen. Gegen diese Externalisierung der mühevollen Arbeit am Content ist ein emphatischer Sammlungs- und Bibliotheksbegriff überfällig.

Schlüsselwörter: Sammlung, Fachinformationsdienst, Digitale Transformation, Natural User Interface, Mobiles Internet, Zukunft der Bibliothek

\section{The Value of Collecting and the Added Value of the Digital: some Scattered Observations on the Current State of Libraries}

Abstract: It is time for the library's renaissance as collection, especially in the digital age. The various user scenarios of digital content require - quite traditionally - its ownership and permanent availability. With respect to the idea of collection, printed, digital and multimedia content have more in common than the current dichotomies of the virtual and the real want to make us believe. Today, if we talk about "Collections", we cannot be silent about "Specialised Information Services" as an arcanum of an understanding of the library that is entirely committed to current needs. At closer view, this turns out to be a strategy of easing the burdens that come with a collection's demands and expectations. An emphatic notion of collection and library is overdue to work against this externalization of the arduous work with the content.

Keywords: Library collection,specialised information services, digital transformation, natural user interface, mobile web, future of the library

„Das unterscheidet [...] die Sortimentsbuchhandlung von der Bibliothek wie von der bibliophilen Kollektion: Dort kommen die Dinge und gehen auch wieder, hier aber bleiben sie, nachdem sie gekommen sind. Dort bedeutet Anwesenheit einen zeitweiligen Aufschub des Verschwindens, hier ist sie der unbefristete Aufenthalt. Stau im Durchfluss versus permanente Präsenz."

Manfred Sommer: Sammeln. Ein philosophischer Versuch 


\section{Einleitung: Vom Unbehagen in der Bibliothek}

Die folgenden Überlegungen zur aktuellen Verortung der Bibliothek in der Umbruchzeit digitaler Transformation sind Ausdruck eines wachsenden Unbehagens. Dieses entspringt der gefühlten Unterbestimmtheit der Selbstreflexion und der Selbstverständigung über die gewandelten Handlungsbedingungen und Zukunftsaufgaben bibliothekarischen Handelns. Bibliotheken befinden sich in einer Umbruchsituation, diese scheint jedoch - von der gängigen Betonung der "Proaktivität" gegenüber den „Herausforderungen“ des Digitalen in Festreden und Grußworten - nicht wirklich in ihrer Radikalität wahrgenommen zu werden.

Genauer gesagt: wahrgenommen nur in der Außen-, nicht aber in der Innenperspektive. Aus der Sicht des nicht-bibliothekarischen Betrachters erscheint die Zukunftsfähigkeit der Bibliothek, die traditionell wie keine andere Institution als Inbegriff materialisierter Kultur und Erkenntnis gilt, zumindest zweifelhaft. Während die Journalistin Inge Kloepfer einen „irren Boom der Bibliotheken“ wahrnimmt ${ }^{1}$ (und es damit zur meistzitierten Referenz verbandspolitischer Repräsentanten gebracht hat), gibt sich die Publizistin Kathrin Passig überzeugt: „Bibliotheken sind Papiermuseen. Wenn sie sich nicht bald grundsätzlich wandeln, haben sie kaum noch eine Existenzberechtigung. Was sie können, kann das Internet besser. ${ }^{2}$ Derart ins Grund- und Gegensätzliche gehende Aussagen sind symptomatisch für die mit der Digitalisierung unserer Lebenswelt einhergehenden Umbruchsituationen. Das Unsichere, Disruptive und radikal Zukunftsoffene, das diese Umbrüche kennzeichnet, lädt geradezu ein zu dystopischen ebenso wie zu utopischen Prognosen.

Allerdings scheint dies nicht für die Innenperspektive, also die Selbstwahrnehmung der Bibliotheks"Community“, zu gelten. Das Digitale wird hier primär als Aufgabenzuwachs, nicht aber als ein zu radikaler Neuorientierung zwingender Zeitenbruch wahrgenommen. Auf dem Nürnberger Bibliothekartag 2015 etwa gab es keine einzige Session oder Podiumsdiskussion zur Zukunftsverortung der Bibliothek in der digitalen Welt, vielmehr wird das Digitale zumeist rückbezogen und damit "eingehegt" in traditionelle Themen bibliothekarischer Arbeit: eine der lebhaftesten Diskussionen entbrannte zum Beispiel um die zukünftige Rolle der verbalen Sacherschließung im Kontext cloudbasierter Discovery-Systeme.

Die folgenden Überlegungen versuchen, der Irritation über diese alles andere als selbstverständliche Selbstgewissheit (wenn nicht sogar Selbstzufriedenheit) in Zeiten objektiver Ungewissheit Ausdruck zu verleihen. Hierbei soll für diesmal nicht das bibliothekarische Handeln - gleichsam extern - von den Zukunftsherausforderungen der digitalen Transformation her hinterfragt werden. Dies ist anderenorts ausführlich diskutiert worden, vor allem mit Blick auf die Ablösung primär textuell 
bestimmten Wissens durch das Modell eines ins Unendliche vernetzten, multimedialen und dynamischen Wissensraumes. ${ }^{3}$

Vielmehr wird bewusst zunächst die Binnenperspektive bibliothekarischen Handelns eingenommen und mit Blick auf zwei prominente Arbeitsfelder entfaltet: die Bestandsentwicklung im Sinne des Aufbaus einer gedruckten und digitalen „Sammlung" einerseits, und die Entwicklung digitaler Mehrwertdienste, mit denen die Zukunftsfähigkeit unserer „Branche“ gesichert werden soll, andererseits. Die Darlegungen sind dabei - schon mit Blick auf die Disruptivität des digitalen Wandels - zwangsläufig nicht systematisch angelegt, sondern als verstreute Bemerkungen zur gegenwärtigen Lage der „Bibliothek“. Gemeinsam ist innen allenfalls ein Grundunbehagen darüber, dass gerade der fortschrittliche, die Transformationsschübe des Netzes „proaktiv" begleitende oder immer schon links überholt habende Bibliothekar gar nicht mehr Bibliothekar im emphatischen Sinne sein will, sondern allenfalls noch ein digitaler Wissenschaftspropädeut oder Hilfswissenschaftler im virtuellen Raum.

Im wissenschaftspolitischen Jargon klingt das dann zumeist so: ,Die Bibliothek als Informationsinfrastruktur unterstützt die gesamte Wertschöpfungskette wissenschaftlichen Arbeitens mit offenen digitalen Inhalten und anspruchsvollen Werkzeugen wissenschaftlichen Kommunizierens, Kollaborierens und Publizierens.' Das hört sich nicht nur nicht besonders sexy an, es kann auch als Indiz dafür gelesen werden, dass das ursprüngliche Selbstverständnis der Bibliothek als die dem Sammeln verpflichtete "Gedächtnisinstitution" (die im übrigen gar nicht radikal genug digital sein kann!) zusehends aus dem Bewusstsein ihrer Protagonisten verschwindet: die Bibliothek als Garant der Einheit von Herkunft und Zukunft, als institutionelle Verschränkung von Erinnerung und Erwartung und damit als eine Basisfunktion der modernen Welt. Einige Bemerkungen zu diesem Bedeutungsverlust des Bibliothekarischen, der auch als Entlastungshandeln gedeutet werden kann, runden daher diesen Beitrag ab.

\section{Sammeln: Vom Content zum Service}

Die „Sammlung" als jahrhunderte-, ja jahrtausendelang grundlegende Kategorie bibliothekarischen Handelns wird hier ganz pragmatisch als systematisch angelegter, fachlich oder thematisch strukturierter Wissenskorpus verstanden. Zum Kernverständnis des Sammelns gehört seit jeher die Eignerschaft und damit die volle Verfügungsgewalt über die gesammelten und aufbewahrten Materialien und Dokumente. Unter der Bezeichnung "Sondersammelgebiete" blieb dieses Sammlungsverständnis auch nach dem zweiten Weltkrieg noch für mehr als sechzig Jahre die Leitidee einer kollektiven, auf wechselseitiger Solidarität beruhenden, von der Deutschen 
Forschungsgemeinschaft kontinuierlich unterstützten Gemeinschaftsleistung zahlreicher deutscher Bibliotheken mit dem Ziel des Aufbaus einer verteilten nationalen Forschungsbibliothek. Leitender Gedanke war hierbei die Maxime eines umfassenden, möglichst vollständigen Bestandsaufbaus in geteilter Verantwortung sowie die überregionale Bereitstellung dieses Bestandes durch Fernleihe und Dokumentlieferung.

Mit der sukzessiven Einstellung dieses Systems seit 2012 wird auch die Frage nach dem „Ende der Sammlung" neu gestellt. Entsprechend formulieren Peter Strohschneider und Christoph Kümmel in ihrem gleichnamigen Breitrag die offizielle Sicht der DFG-Verantwortlichen: „Das Aufgabenspektrum von Fachinformationsdiensten und der Charakter von Sammlungen schließen sich zwar keineswegs aus, gleichwohl kann ein traditioneller Sammlungsbegriff in diesem Zusammenhang auch unangemessen und irreführend sein. Denn vielfach beziehen sich die Informationsbedürfnisse der Fächer nicht mehr auf einen lokal gepflegten Buchbestand, sondern zunehmend auf bestandsunabhängige digitale Informationen“. ${ }^{4}$

Diese Aussage ist in ihrer Verengung des Sammlungsverständnisses auf den vor Ort vorgehaltenen Bestand gedruckter Materialien beim besten Willen nicht nachvollziehbar: Die bloße Faktizität, dass Bibliotheken sich seit ihren Anfängen primär mit der Sammlung handgeschriebener oder gedruckter Dokumente beschäftigen, und erst seit einem guten Vierteljahrhundert auch auf digitale Medien ausgreifen (von denen es zuvor nun einmal nicht besonders viele gab), hat mit dem oben umrissenen, allgemein gebräuchlichen Verständnis von „Sammlung“ nun wirklich nichts zu tun.

Tatsächlich kann eine „Sammlung“ selbstverständlich Inhalte beliebiger Materialität umfassen: digitale und analoge, elektronische und gedruckte, textuelle und multimediale, statische und dynamische Informationsressourcen. Auch der Grad ihrer Zugänglichkeit und damit „Offenheit" für den Nutzer war schon immer und wird auch in Zukunft heterogen sein: ein gedrucktes Pflichtexemplar kann je nach Rechtsgrundlage nur im Lesesaal zugänglich oder unter bestimmten Konditionen auch für die Ausleihe zugelassen sein, die Lizenz einer elektronischen Zeitschrift mag ihre Lektüre auf die eingeschriebenen Nutzer der lizensierenden Institution begrenzen oder für eine ganze Region öffnen, sie mag dauerhafte Archivrechte, das Recht zur Weiterverarbeitung und Distribution beinhalten oder ausschließen, usw. usw. Die jeweilige Granularität der Offenheit eines Content-Corpus kann also ebenfalls wohl kaum den Zweifel an der Zukunftsfähigkeit des Sammlungskonzepts motivieren, wie er im oben zitierten Beitrag artikuliert wird, wenn auf die vermeintlich „lokale“ Bindung eines „Buchbestandes“ abgestellt wird. 
Überblickt man die intensiv und kontrovers geführten Debatten um die Verabschiedung des Sondersammelgebietsprogramms zugunsten von Informations"diensten", die primär durch die jeweils aktuellen Bedürfnisse der Fachwissenschaft getriggert sind, wird rasch klar, worum es bei diesem Wechsel des Förderparadigmas geht: Die Verantwortung für den Auf- und Ausbau eines signifikanten Bestandes (noch einmal: gleichgültig ob gedruckt oder digital), für seine Pflege und Bewahrung und damit seine Verfügbarkeit für eine unabsehbare Zukunft soll grundsätzlich aus dem Verantwortungsbereich des Förderhandelns externalisiert werden: Es geht im Kern um die Selbstentlastung der Deutschen Forschungsgemeinschaft wie auch der antragstellenden Bibliotheken von der Verpflichtung auf „Inhalte“ und Wissensbestände zugunsten von Diensten der Bereitstellung und des Zugriffs, die „on top“ dieser Inhalte operieren. „Für das Verständnis der Neuausrichtung der Förderung ist wesentlich“, so Strohschneider und Kümmel, „dass die Fachinformationsdienste ausdrücklich auf eine Optimierung des Zugriffs auf Informationen abzielen - sei es auf gedruckte Bücher oder digitale Medien - nicht aber vorrangig auf deren umfassende Beschaffung und Archivierung. [...] Ein funktionierendes Gesamtarchiv von Forschungsliteratur und Forschungsmaterialien [...] ist aus dieser Perspektive vielmehr eine kooperativ wahrzunehmende Aufgabe der Nationalbibliotheken und -archive oder vergleichbarer Einrichtungen nationalen Rangs rund um den Globus. ${ }^{\prime 5}$

Dass damit - bezogen auf den Content - auch eine massive finanzielle Entlastung verbunden ist, versteht sich von selbst, und wird besonders deutlich, wenn man noch einmal auf den Kern des Sammlungsbegriffs blickt. Inhaltlich geht es beim Sammeln natürlich immer um das Systematische und Strukturierte des Bestandsaufbaus entlang fachlicher oder materialspezifischer Schwerpunkte. Eine international ausgerichtete, geisteswissenschaftliche Forschungsbibliothek sammelt eben anders als eine wirtschaftswissenschaftliche Fachbibliothek. So archiviert beispielsweise die Bayerische Staatsbibliothek nicht alle möglichen wissenschaftlichen Websites, sondern nur diejenigen, die in ihr Sammlungsprofil passen (u.a. zur Musik, Osteuropaforschung und Geschichte). Formal bedeutet - und das ist hier entscheidend - die Tätigkeit des Sammelns aber immer und unausweichlich die Übernahme einer langfristigen Ressourcenverantwortung für die Sammlungsgegenstände: Bücher werden gekauft und auf Dauer magaziniert, digitale Objekte und Datenbestände lizensiert, für den dauerhaften Zugriff gehostet und langzeitverfügbar archiviert, "offene", frei im Netz angebotene Inhalte gespeichert, migriert und emuliert (Stichwort: Preservation Planning und Action).

Das ist ressourcenintensiv, und zwar im Regelfall - entsprechend der im Sammlungsgedanken stets mitgemeinten Nachhaltigkeit und Dauerhaftigkeit - in Form langfristig indisponibler Fixkosten. So 
belaufen sich allein die Speicher- und Archivierungskosten der Bayerischen Staatsbibliothek für ihren gesamten, kontinuierlich wachsenden digitalen Datenbestand mittlerweile pro Jahr auf rund 500.000 Euro, und den Einnahmen aus Dokumentlieferung und Fernleihe ihrer Sammlungsbestände stehen Prozesskosten in dreifacher Höhe gegenüber. Denn immer geht es beim Sammeln (des Digitalen wie Gedruckten) um den Besitz, das Eigentum am Sammlungsinhalt im Interesse seiner „immerwährenden“ Verfügbarkeit und Verwendbarkeit in gegenwärtig oft noch gar nicht absehbaren Nutzungsszenarien. „Zugleich wird deutlich“, so Andreas Degkwitz, „von welcher Bedeutung für Bibliotheken Besitz oder Eigentum der gesammelten Materialien sind, um sie aufbereiten, verarbeiten und verlinken und damit wiederverwendbar zur Verfügung stellen zu können. ${ }^{16}$

Diese „Ressourcenpflichtigkeit" des Sammelns war im alten System der Sondersammelgebiete in zweifacher Hinsicht zumindest ein Stück weit abgefedert worden: Zum einen durch das im Konzept einer verteilten nationalen Forschungsbibliothek angelegte Solidarprinzip, zum anderen durch das bewusst auf Langfristigkeit berechnete Förderhandeln der Deutschen Forschungsgemeinschaft. Beides wird im neuen System explizit kassiert. Die Förderung der neuen Fachinformationsdienste ist kompetitiv angelegt, sie müssen im Dreijahresturnus immer neu begründet und gegebenenfalls gegen konkurrierende Mitbewerber neu erkämpft werden: „Eine Projektförderung", so Strohschneider und Kümmel, „die durch die Ermöglichung stetiger Fortsetzungsanträge einen quasiinstitutionellen Charakter annimmt, ist dabei ausdrücklich nicht die angestrebte Lösung. ${ }^{77}$ Das Zerbrechen des auf dem Prinzip des wechselseitigen Geben und Nehmens aufbauenden traditionellen Sammlungssystems wird dabei bewusst in Kauf genommen: In den ersten zwei Förderrunden sind eine Reihe von Fächern entweder durchgefallen oder gerade im Sammlungsbereich rabiat beschnitten worden, oder sie sind erst gar nicht mehr angetreten.

Damit ist derzeit unter anderem weder für die Philosophie noch für die Psychologie eine Schwerpunktbibliothek in Deutschland vorhanden. Und auch für die große, in 2015 laufende dritte und den ersten Förderturnus dann für sämtliche ehemaligen Sondersammelgebiete abschließende Antragsrunde gelten selbstverständlich die mahnenden (und eigentümlich umwegig formulierten) Worte der DFG-Verantwortlichen: „In jedem Fall besteht in der neu ausgerichteten Förderung ein deutlich erhöhter Anspruch für die Begründung eines vorsorgenden Bestandsaufbaus in den einzelnen Vorhaben. Die absehbare Nutzungshäufigkeit des Bestandes wird dabei zwar nicht per se ein Argument für oder gegen den Aufbau und die Pflege einer Sammlung sein. Je nach den Forschungsinteressen der Fachcommunity kann sie bei der Bewertung der Relevanz einer Sammlung dennoch eine entscheidende Rolle spielen, wenn es um die Verteilung der Mittel geht. ${ }^{18}$ Und die 
Leiterin der DFG-Gruppe „Wissenschaftliche Literaturversorgungs- und Informationssysteme“ geht noch einen Schritt weiter, wenn sie jede langfristig angelegte, wie auch immer "komplementäre“ Verantwortung für die wissenschaftliche Informationsversorgung zurückweist: „Die Rolle der DFG im Gesamtsystem der wissenschaftlichen Informationsinfrastrukturen ist es, Anreize zu setzen, Startkapital bereit zu stellen, fachliche und strukturelle Entwicklungen zu stimulieren. Ihre Rolle ist es nicht, die einmal angestoßenen Entwicklungen und die entstandenen Strukturen dauerhaft zu finanzieren. ${ }^{\prime 9}$

Diese Erklärung der Nicht-Zuständigkeit impliziert jedoch zwangsläufig eine sehr weitgehende Einforderung, wenn nicht gar Überforderung derjenigen Einrichtungen, die die oben bezeichnete langfristige Ressourcenverantwortung für die von ihnen gepflegten Sammlungen übernommen haben. Sie können sich zukünftig weder auf das Solidaritätsprinzip „aller anderen“ Instanzen im Gesamtsystem der Informationsversorgung mehr verlassen noch auf die Bereitschaft der maßgeblichen nationalen Förderinstitution zur verlässlichen Unterstützung der verteilten Infrastrukturen. Strukturell geraten die sammlungsführenden Bibliotheken damit in die gleiche Rolle, in der sich heute Netzprovider wie Telekom und AT\&T sowie Hersteller mobiler Devices wie Samsung oder Huawei gegenüber Facebook und Co. befinden. Während sich letztere immer tiefer in den digitalen Lebenswelten ihrer Nutzer verankern, sind erstere auf das „verborgene“ und weit weniger lukrative Backbone-Geschäft mit Netzen und Hardware beschränkt, das andererseits aber die unentbehrliche Basis aller Endnutzerdienste ist: „Wenn Facebook es schafft, wie jetzt für eine Kooperation mit dem Handy-Fabrikanten HTC angekündigt, eine Schicht über das komplette Handy zu legen, dann würden die Hersteller kaum noch unterscheidbar sein. Und die Mobilfunkprovider endgültig in der Rolle des bloßen dummen Dienstleisters. ${ }^{\text {"10 }}$

Genau dies ist aber auch das implizite "Geschäftsmodell“ des neuen Systems der Fachinformationsdienste: Während im „Frontoffice“ antragserfolgreiche Einrichtungen, zusehends entlastet von der Verantwortung für den Content, schicke Services im Kontext virtueller Forschungsumgebungen aufbauen ${ }^{11}$, dürfen sich die ganz altmodisch „einem Gesamtarchiv von Forschungsliteratur und Forschungsmaterialien“ verpflichteten Institutionen im „Backoffice“ mit der ressourcenintensiven Aufgabe des Bestandsaufbaus, seiner Erschließung, Magazinierung, Bereitstellung und Langzeitarchivierung herumschlagen. Sie sind damit in der Tat in der Rolle des dummen Dienstleisters.

Dass dieses Modell nicht funktionieren wird, liegt auf der Hand. Denn im Unterschied zu Netzprovidern und Hardwareproduzenten, die eben nicht Facebook „können“, können die großen, 
dem Sammlungsgedanken verpflichtet bleibenden Bibliotheken durchaus auch die Dienste im Frontend. Gerade sie können ihr Alleinstellungsmerkmal, eben die großen und stetig weiter wachsenden Sammlungen (digital und gedruckt), in forschungsangemessenen Diensten ausspielen, während umgekehrt gilt: Dienste ohne Inhalte sind leer und für Wissenschaft und Forschung uninteressant. Ein Indiz dafür ist, dass etwa die Bayerische Staatsbibliothek in jüngster Zeit immer wieder angefragt wird, ob sie nicht ihre Sammlungsaktivität gerade in den Feldern der aus dem DFGFördersystem herausgefallenen Fächer Philosophie und Psychologie signifikant verstärken könne natürlich aus eigenem „Budget“.

Ein weiteres Indiz für das durchaus vorhandene Bewusstsein von der "Content-Pflichtigkeit" moderner Informationsinfrastrukturen, das zugleich den potenziellen, künftigen Umgang mit der geschilderten Problematik vorzeichnet, ist die erweiterte Richtlinie 2013/37/EU des europäischen Parlaments und des Rates vom 26. Juni 2013 über die Weiterverwendung von Informationen des öffentlichen Sektors. Grundsätzlich auf die weitgehende Öffnung von digitalen Datenbeständen in öffentlicher Hand für ihre kommerzielle Nachnutzung angelegt, trägt gerade diese Richtlinie dezidiert der Einsicht Rechnung, dass die von Bibliotheken, Archiven und Museen digitalisierten Datenbestände nicht einfach im Netz "da“ sind, sondern in ihrer Herstellung und langfristigen Verfügbarhaltung einen permanenten und intensiven Ressourceneinsatz verlangen. Entsprechend konzediert die Richtlinie den contentführenden Kultureinrichtungen nicht nur die Gebührenerhebung für die mit der unmittelbaren Bereitstellung von Inhalten einhergehenden Grenzkosten, sondern auch für die mit der Vorhaltung und Archivierung dieser Inhalte verbundenen Infrastrukturkosten, sogar zuzüglich einer angemessenen Gewinnspanne, die der Abgeltung der "Grundlast" der Betriebsbereitschaft der Kulturinstitutionen dient: „In Bezug auf Bibliotheken, Museen und Archive und angesichts ihrer Besonderheiten könnten die Gebühren, die im Privatsektor für die Weiterverwendung identischer oder ähnlicher Dokumente erhoben werden, bei der Ermittlung der angemessenen Gewinnspanne berücksichtigt werden. ${ }^{\text {“12 }}$

Angesichts der absehbaren Selbstentlastung zunehmend weiterer Teile der Bibliothekslandschaft und ihrer maßgeblichen Fördereinrichtung von der Solidarverpflichtung einer verteilten Sammlungsverantwortung für Wissenschaft und Forschung spricht wenig dagegen, derartige Refinanzierungsoptionen zumindest dort, wo nicht gesetzliche Pflichtaufgaben dem entgegenstehen, im Interesse eines Lastenausgleichs zwischen Contentinhabern und Diensteanbietern auch im öffentlichen Sektor in Erwägung zu ziehen. Diese Option ruft das eigentlich Selbstverständliche in Erinnerung: auch im Digitalen sind Wissensgüter nicht einfach „offen“ und kostenfrei abgreifbar, sie sind dies nur scheinbar, da eben ein "anderer" in die Ressourcenverantwortung gezogen wird. Der 
Vorsitzende des Verbands der Historiker und Historikerinnen Deutschlands, Martin Schulze Wessel hat im Beitrag „Sammeln für die Interessen von morgen“ in der Frankfurter Allgemeinen Zeitung den Umstieg auf das neue System der Fachinformationsdienste als „Gestus der forcierten Modernisierung" bezeichnet. ${ }^{13}$ Ergänzen könnte man, das dieses System im Kern eine FreeloaderMentalität befördert, die auf die Selbstentlastung von der anstrengenden, auf einen sehr langen Atem berechneten und scheinbar ganz und gar unmodernen Sammlungsaufgabe der Bibliothek zielt.

\section{Digitale Mehrwertdienste: Content in Context}

Angesichts der digitalen Transformation lassen sich die bisherigen Ausführungen, nimmt man die Perspektive der Contentinhaber ein, nun aber durchaus auch ins Positive wenden: Wer die niemals endende Fron des Sammelns auf sich nimmt und es zugleich versteht, diese Wissensgüter in den neuen und vielfältigen Nutzungsszenarien der digitalen Lebenswelt zu aktivieren und zu kontextualisieren, hat - die Prognose darf gewagt werden - eine gute Zukunft vor sich.

Der unmittelbare Nutzwert digitalisierter und genuin digitaler Sammlungen liegt natürlich in der Betrachtung über sogenannte "Viewer" als Benutzerschnittstelle auf diversen Plattformen und Portalen im Web (z.B. institutionelle Webauftritte, regionale Kulturportale, Deutsche Digitale Bibliothek, Europeana, Archivportal-D). Das Scannen und Bereitstellen im Netz sind jedoch nur die ersten Schritte der digitalen Transformation. Ihr Nutzen besteht in der Zugänglichkeit für alle, zu jedem Zeitpunkt und von jedem Internetanschluss weltweit. Die hier zum Einsatz kommenden Techniken und Workflows sind mittlerweile gut beherrscht, eingespielt und fast schon nicht mehr der Rede wert - auch wenn der fortschreitende Digitalisierungsprozess tradierter, „analoger“ Kultur- und Wissensobjekte gewiss noch Jahrzehnte dauern wird.

Der eigentliche Mehrwert des Digitalen erschließt sich jedoch erst dann, wenn der digitale Content in die neuen, technikgetriebenen Nutzungsszenarien des Netzes integriert wird. Gemeint sind Applikationen, die die Digitalisate in kulturellen, schulischen, wissenschaftlichen, wirtschaftlichen und touristischen Anwendungsumgebungen „arbeiten“ lassen und so Nutzungserfahrungen und erlebnisse ermöglichen, die im Analogen nicht gegeben sind. Es geht also um die Kontextualisierung der digitalen und digitalisierten Inhalte, gemäß der Formel: „Content is King, Context is Queen“.

An erster Stelle ist hier die semantische Verknüpfung der digitalen Objekte über „Linked Data" zu nennen, wodurch jedes digitale Objekt sparten- und fachübergreifend mit anderen Objekten vernetzbar wird. Auf diesem Wege erschließen sich kulturelle Kontexte über einzelne Archive, Museen, Bibliotheken und Wissenschaftseinrichtungen hinweg, es entstehen themenbezogene 
Contentcluster für wissenschaftliche Fragestellungen ebenso wie zum Beispiel für virtuelle Ausstellungen. Weiterhin spielt die Öffnung der Daten - der Digitalisate selbst wie ihrer Metadaten zur freien Nachnutzung als „Open Data“ eine wesentliche Rolle. Damit ändert sich - bezogen auf ihre digitalen Angebote - fundamental die Rolle der Kultur- und Wissenschaftsinstitutionen: vom Bereitstellen des Zugangs zum Bereitstellen hochwertiger, originaler digitaler Masterdateien, die entweder außerhalb oder innerhalb der technischen Infrastrukturen der Kultureinrichtungen vernetzt, angereichert, wieder- und weiterverwendet werden. Als öffentliche Einrichtungen garantieren die Kultur- und Wissenschaftseinrichtungen zudem für die Vertrauenswürdigkeit und langfristige Integrität der Daten, ein wichtiges Alleinstellungsmerkmal gegenüber vielen kommerziellen Angeboten.

Hierzu zwei Beispiele aus der Bayerischen Staatsbibliothek: „DaFo“ (Daten für die Forschung) ist eine Self-Service-Plattform für Wissenschaftler zum freien Download hochauflösender Digitalisate und Volltexte (bis zu 1.000 Werke pro Vorgang). Zukünftig soll diese Plattform auch zur Analyse von TextMassendaten im Sinne des Data Mining durch die Digital Humanities ausgebaut und damit zum direkten Arbeiten in der Plattform selbst geöffnet werden. Einen Schritt weiter geht das von der Bayerischen Staatsbibliothek in einem globalen Konsortium mitgestaltete „International Image Interoperability Framework" (IIIF). Diese Plattform erlaubt es dem Nutzer, an verschiedenen Institutionen (die weltweit verteilt sein können) aufbewahrte Digitalisate virtuell zusammenzuführen, direkt im eigenen Browser zu laden und in einer Mehrfensteransicht zu betrachten und zu bearbeiten (Kommentare, Annotationen etc.). So können verstreut in der Welt aufbewahrte Objekte, wenn sie einmal digitalisiert sind, virtuell zusammengeführt werden: Der Nutzer wird zum Kurator seiner eigenen, persönlichen Sammlung.

Diese Beispiele zeigen bereits deutlich den „Mehrwert“ des Digitalen: Die Produktion (Scanning) und die Bereitstellung eines Zugangs zu Digitalisaten ist allenfalls der Startpunkt. Jetzt stellt sich die Aufgabe, die Digitalisate (gleich welchen Medientyps) aktiv zu verbreiten und die Nutzer selbst eigenständig damit und daran arbeiten zu lassen. Die partielle Lösung des Digitalisats vom Ursprungsort seiner Aufbewahrung und Bereitstellung wird hierbei bewußt in Kauf genommen. In der Fernperspektive zeigt sich hier ganz praktisch die globalisierte Entgrenzung der eigenen, immer partiellen Sammlung in einer digitalen Weltbibliothek.

Neben die beschriebene "Öffnung" und Vernetzung der digitalen Bestände tritt gleichwertig ihre Einbindung in spezifische Applikationen, die den State-of-the-Art technologischer Möglichkeiten voll 
ausschöpfen und diese Bestände zu innovativen digitalen Informationsprodukten formen. Die derzeit wichtigsten Anwendungsbereiche sind:

1. Mobile Apps für Tablets und Smartphones: Der Maßstab, nach dem digitale Datenbestände im mobilen Internet strukturiert, gefiltert und aufbereitet werden, ist ihre „situative Passung": Digitales wird möglichst exakt mit Bezug auf die Situation designed, in der ich mich als Nutzer gerade befinde, und mit Bezug auf das Nutzungsinteresse, das ich in dieser Situation aktuell habe. Prägnante und sehr erfolgreiche Beispiele sind die Location-Based-Service-App „Bayern in historischen Karten“ und die Augmented-Reality-App „Ludwig II.“ der Bayerischen Staatsbibliothek. Diese Apps stellen Digitalobjekte passend zum jeweiligen Standort des Betrachters bereit, im Falle von AugmentedReality direkt integriert in das Kamerabild der Realwelt. Ein Grundprinzip derartiger Kultur-Apps ist das oft einrichtungsübergreifende "Highlighting" ausgewählter Segmente aus umfassenderen Sammlungsclustern, so zum Beispiel in der App „Famous Books“ 50 Spitzenstücke aus dem Gesamtangebot der digitalen Sammlungen der Bayerischen Staatsbibliothek.

2. Die Navigation digitaler Inhalte im mobilen Internet ist am Paradigma der komplett über Touchscreen und gestenbasierte Steuerung bedienbaren Endgeräte wie Smartphone, Tablet oder Wearable ausgerichtet. An die Stelle herkömmlicher „Graphic User Interfaces“ (GUI) treten „Natural User Interfaces" (NUI), die das Verhalten von digitalen Objekten in Reaktion auf Sprache, Gestik und Berührung gestalten. ${ }^{14}$ Hierdurch eröffnen sich neue Nutzungsszenarien im digitalen Raum, die diesen zugleich zurückbinden an den „physischen“ Ort einer Sammlung, also zum Beispiel das bestandshaltende Museum. Dies gilt vor allem für die Begleitung von Ausstellungen durch vor Ort installierte Präsentations-Devices, die etwa 3D-Digitalisate von Kulturobjekten zur direkten, haptischen Manipulation im virtuellen Raum bereitstellen.

Derartige Anwendungen sind vor allem auch für die mit Simulationen arbeitenden Wissenschaften zukunftsbedeutsam, wie das "Virtual Reality and Visualisation Centre" (V2C) des Münchner LeibnizRechenzentrums exemplarisch zeigt. Hier ist eine digitalisierte antike Grabkammer virtuell begehbar, ein 3D-Digitalisat einer Terrakottastatue „begreifend" zu studieren, und der berühmte Himmelsglobus des Arboreus in 3D virtuell "drehbar" und bis in die Details des Malprogramms zoombar. Auch hier gilt: Einmal digitalisiert, sind die Zukunftsanwendungen prinzipiell unabsehbar. So bilden die genannten Objekte beispielsweise ein ideales Material für das finanziell niedrigschwellige Head-Mounted-Display "Oculus Rift", das eine den Betrachter vollständig umgebende virtuelle Realität simuliert. Zudem kann mittels preisgünstiger 3D-Drucker der Kreis vom Original zum Digitalisat und zurück zum realen „Simulacrum des Originals“ geschlossen werden mit 
zukünftig neuen Möglichkeiten für Museumsshops und generell die Verwertung digitalisierter Kulturgüter: der Nutzer kann seine eigene Sammlung mit „nach Hause“ nehmen.

3. Die Nutzungsszenarien digitaler Kulturobjekte sind aktuell noch sehr stark textuell bestimmt (z.B. Data Mining in Volltexten), vor allem infolge der Digitalisierungsfortschritte im Bibliotheksbereich. Künftig werden jedoch gerade durch die zunehmende Digitalisierung musealer Sammlungen primär bildorientierte Zugriffe und Bereitstellungsformen dominieren. So bietet etwa die von der Bayerischen Staatsbibliothek gemeinsam mit dem Fraunhofer Heinrich-Hertz-Institut entwickelte "Bildähnlichkeitssuche" (Visual Search) einen völlig neuen Zugriff auf digitale Massendaten: Ausgehend vom jeweils für den Nutzer interessanten Bild (das man auch selbst hochladen kann) werden hinsichtlich Form, Farbe und Strukturmerkmalen ähnliche Bilder aus potenziell unendlich großen Datenbeständen ausgefiltert und angezeigt. Auf diese Weise findet erneut eine Rekontextualisierung der Sammlungen statt.

4. Die digitale Transformation läßt die Kultur- und Wissensgüter nicht „ortlos" werden, sie kann vielmehr die Präsenz und Zugänglichkeit der Originale vor Ort in den Kultur- und Wissenschaftseinrichtungen steigern und unterstützen. Die Bayerische Staatsbibliothek etwa experimentiert derzeit mit sogenannten Beacons. Das sind in der Bibliothek verteilte Signalgeber, die im Zusammenspiel mit einer entsprechenden App eine kontinuierliche Begleitung und Navigation des Nutzers zu vielfältigen Points of Interest im Haus erlauben. Am POI selbst ruft die App dann die passenden digitalen Daten auf. Beacons bieten eine orientierende Navigation in geschlossenen Räumen, wo GPS-Signale im Regelfall versagen, und können so eine komfortable, leicht ausbau- und veränderbare Alternative zu klassischen Audioguides oder QR-Codes sein. Dies wiederum ist ein weiteres Beispiel, wie innovative Technologien digitale Inhalte in vielfältigen, gegenwärtig oft noch unvorhersehbaren Nutzungsszenarien immer wieder neu und immer wieder anders „aktivieren“ können.

Durch die offene Bereitstellung ihrer Digitalisate und deren Einbindung in die beschriebenen Plattformen und Applikationen werden Kultur- und Wissenschaftsinstitutionen Teil einer letztlich globalen Wissensinfrastruktur im Web. Mit Blick auf die beschriebenen Netzwerkeffekte geht es also (1) immer darum, digitalen Content zu haben, und (2) ebensosehr darum, wie gut dieser Content mit der übrigen digitalen Welt verbunden ist. Im Netz löst sich die Identität der Sammlungen nicht auf, sondern kontextualisiert sich neu. 


\section{Fazit: Bibliothek und Moderne}

Für die dem Sammlungsgedanken verpflichtet bleibende Bibliothek kommt dem Besitz an den Wissensgütern ein hoher Stellenwert zu. Die dauerhafte Verfügungsgewalt über die Informationsressourcen ist, wie gezeigt wurde, die entscheidende Voraussetzung für eine Vielzahl von Diensten, gerade solchen, die dem Nutzer das interaktive, anreichernde und verändernde Arbeiten mit den bereitgehaltenen Inhalten erlauben. Die personelle und finanzielle „Ressourcenlast“ des Sammelns wird im Digitalen noch potenziert, denn die Gesamtkosten aller unter dem Begriff der „Data Curation“ zu subsummierenden Maßnahmen übersteigen die Kosten der Magazinierung und Erhaltung gedruckter Bestände oft um ein Vielfaches. Neben Forschungsdaten sind es vor allem die in der Arbeit mit digitalen Inhalten entstehenden Annotationen, Kommentare, Anreicherungen, Veränderungen und Neukontextualisierungen, die ihrerseits Teil der Sammlung werden und wiederum neue Angebote ermöglichen. Auch im Digitalen gilt also die bündige Formulierung Franz Georg Kaltwassers: „Eine Bibliothek ist nur lebendig, wenn sie wächst. “15

Vor diesem Hintergrund wird die Bereitschaft so mancher Einrichtung, sich zu einer digital gewendeten Gebrauchsbibliothek herunter zu pragmatisieren, die nur durch das gerade aktuell Nachgefragte getriggert wird, durchaus verständlich. Die neuen Förderformate bergen hier ein verlockendes Entlastungsversprechen: der tendenzielle Verzicht auf die Pflege und den Ausbau großer Sammlungen erlöst nicht nur von der Last der Ressourcenverantwortung, sondern ebensosehr von der Last und Belastung durch die Sorge um das anvertraute "Gedächtnis" der Menschheit. Denn schließlich materialisieren sich Herkunft und Erfahrung des Menschen in der emphatisch gedachten - Bibliothek: sie ist das Gebäude des Gedachten, mit all seinen Erkenntnissen, geistigen und weltanschaulichen Kämpfen, seinen Irrwegen, Irrtümern und Umwegen, seinen verifizierten und falsifizierten Theorien und seinen (zumindest manchmal auch) ewigen Wahrheiten. Die Verantwortlichkeit für dieses Gebäude muss zwangsläufig dort als Ballast empfunden werden, wo man innovierend und "dienst“leistend, als Lernort, Werkstatt und Kompetenzvermittler ${ }^{16} \mathrm{zu}$ neuen bibliothekarischen Horizonten aufbrechen möchte.

Eine dem Sammlungsgedanken verpflichtet bleibende Bibliothek bewegt sich dagegen unhintergehbar im Spannungsfeld von Vergangenem und Zukünftigem, von tradiertem Wissensgut und seiner Aktivierung für neue Erkenntnis. Damit trägt sie zugleich unauslöschlich die Signatur der Moderne, die Joachim Ritter in seinem epochemachenden Essay „Hegel und die französische Revolution“ als „Entzweiung von Herkunft und Zukunft" ${ }^{117}$ begriffen hat: „Das Wesen der modernen politischen Revolution [...] liegt für Hegel [...] in der Setzung von Ordnungen, die ihrem Prinzip nach voraussetzungslos wie ein radikaler Neuanfang, dem nichts vorausgehen soll, alles Vorgegebene, 
Geschichtliche und Überlieferte von sich ausschließen." ${ }^{18}$ Odo Marquard hat dieses Entzweiungstheorem aufgenommen und zu einer generellen Diagnose der modernen Lebenswelt verdichtet: „Die moderne Welt beginnt dort, wo der Mensch methodisch aus seinen Traditionen heraustritt: wo sich seine Zukunft aus seiner Herkunft emanzipiert. ${ }^{\text {“19 }}$ Angesichts der Dialektik von Herkunft und Zukunft ist die Frage nach der Entstehung des Neuen für Marquardt allerdings wie folgt zu beantworten: „Mir scheint nun eine der wichtigsten Antworten auf die Frage - wie ist Neues überhaupt menschenmöglich? - diese zu sein: nicht ohne das Alte. Das ist hier meine These, die sich auch folgendermaßen formulieren lässt: Zukunft braucht Herkunft. ${ }^{20}$

Es ist unschwer zu erkennen, dass die Bibliothek, sofern und insoweit sie Sammlung ist, eine Instanz (wenn nicht die maßgebliche) unserer Kultur ist, die genau diese Vermittlungsleistung bewältigt. Im auswählenden Erwerben, Ordnen, Erschließen, Indexieren und Bewahren bekommt sie das stetig wachsende Wissensgebirge des Herkünftigen in den Griff und stellt es für den weiteren Ausgriff ins Zukünftige zur Verfügung. Im oft zitierten Kreislauf „Aus Forschung wird Bibliothek, aus Bibliothek Forschung" ist sie damit die entscheidende Aktivierungsinstanz des Wissens: Im bibliothekarischen (im Gegensatz zum bibliophilen) Sammeln wird das Gesammelte nicht dem toten Gedächtnis überantwortet, sondern aktiviert für seine zukünftige Nutzung, Entwicklung und auch Überwindung.

Dieser emphatische Sammlungsbegriff gilt auch und gerade im Feld digitaler Ressourcen: der Zugriff auf die vernetzte Datenwelt in Form ihrer Durchdringung mit Kommentaren, Annotationen, Verlinkungen, Anreicherungen, Kontextualisierungen und Nachnutzungen, deren Manifestationen wiederum Teil des Sammlungsgeschehens werden, setzt immer die dauerhafte und verläßliche Verfügungsgewalt über die Inhalte voraus. Das Grundmotiv bibliothekarischen Sammeln bleibt im Analogen wie im Digitalen identisch: Es ist die vermittelnde Bewegung des im „Gestern“ Erreichten hin zu seiner Überschreitung ins "Morgen“, anders gesagt: die dauerhafte Ermöglichung der Auseinandersetzung mit schon Gedachtem, Erfahrenem, Erprobtem und "Gewusstem“ im Interesse seiner Prüfung, hermeneutischen Befragung, empirischen Falsifizierung und dialektischen Überwindung durch das Neue.

Gerade dieses emphatische Verständnis der „Bibliothek“ als Instanz der Vermittlung von Herkunft und Zukunft, Erfahrung und Erwartung lässt sich als Hintergrundmodell des Systems der vorsorgenden, umfassenden und auf Vollständigkeit zielenden Sammlung für eine unabsehbare Zukunftsnutzung identifizieren. Und gerade darum wiegt es schwer, wenn dieses der Moderne zutiefst eingeschriebene Konzept nun einer vom bloß Gegenwärtigen her determinierten „Nachfrage- und Nutzerorientierung“ ${ }^{21}$ geopfert wird: „Welche Themen künftig relevant sein 
werden, wissen wir nicht. Nur die formalen Auswahlprinzipien der alten Sondersammelgebiete erlauben es, Literatur vorsorglich zu erwerben. Die Dynamik in den Geisteswissenschaften hängt paradoxerweise von statischen Bedingungen ab: von der Verlässlichkeit und Vollständigkeit der Literaturversorgung - von der nun aus den DFG-Richtlinien verbannten Tätigkeit des Sammelns. ${ }^{\text {"22 }}$

Es ist ersichtlich, dass dies keineswegs ausschließlich ein Thema der Geisteswissenschaften, inklusive der Digital Humanities ist, sondern ganz grundsätzlich der reflektierten Selbstvergewisserung der Aufgabe von „Bibliothek“ in der modernen Welt. Eine Rückbesinnung auf diese Aufgabe sollte spätestens dann einsetzen, wenn auch das neue System in einigen Jahren seiner allfälligen „Evaluierung“ unterzogen wird. Natürlich darf man sich nicht der naiven Erwartung einer weitgehenden „Revision“ der aktuellen Praxis hingeben. Aber es muss schon daran erinnert werden, dass hier mehr auf dem Spiel steht als eine „Optimierung des Zugriffs auf Informationen“23.

\section{Dr. Klaus Ceynowa}

Bayerische Staatsbibliothek

Ludwigstrasse 16

D-80539 München

ceynowa@bsb-muenchen.de

\footnotetext{
${ }^{1}$ Kloepfer, Inge: Der irre Boom der Bibliotheken. In: Frankfurter Allgemeine Sonntagszeitung, Nr. 11, 16. März 2014.

http://www.bibliotheksverband.de/fileadmin/user upload/DBV/ueber uns/vorstandsvorträge/d $2014 \quad 03 \quad 16$ FAS Der irre Boom der Bibliotheken.pdf

2 Passig, Kathrin: Die Zukunft des Papierverleihs. In: Zeit-Online, 4. November 2013. http://www.zeit.de/digital/internet/2013-11/passig-bibliotheken-internet

${ }^{3}$ Vgl. Ceynowa, Klaus: Der Text ist tot. Es lebe das Wissen. In: Hohe Luft. Zeitschrift für Philosophie 1 (2014), S. 53-57. Ceynowa, Klaus: Digitale Wissenswelten - Herausforderungen für die Bibliothek der Zukunft. In: Zeitschrift für Bibliothekswesen und Bibliographie 61 (2014), S. 236-239.

4 Kümmel, Christoph; Strohschneider, Peter: Ende der Sammlung? Die Umstrukturierung der Sondersammelgebiete der Deutschen Forschungsgemeinschaft. In: Zeitschrift für Bibliothekswesen und Bibliographie 61 (2014), S. 120-129; 128.

${ }^{5}$ Kümmel; Strohschneider (Anm. 4) S. 125 und 127.

${ }^{6}$ Andreas Degkwitz: Digitale Sammlungen. Vision eines Neubeginns. In: Bibliothek. Forschung und Praxis 38 (2014), S. 415.

${ }^{7}$ Kümmel; Strohschneider (Anm. 4) S. 124.

${ }^{8}$ Kümmel; Strohschneider (Anm. 4) S. 128.

${ }^{9}$ Lipp, Anne: Wissenschaftliche Informationsinfrastrukturen und Forschungsförderung: Impulse und Strategien im Strukturwandel. In: Zeitschrift für Bibliothekswesen und Bibliographie 61 (2014), S. 280-287; 284.

${ }^{10}$ Kuhn, Johannes; Martin-Jung, Helmut: Hauptsache, es bleibt in der Familie. In: Süddeutsche Zeitung, Nr. 72, 27. März 2015.

${ }^{11}$ Deren Nachfrage seitens der Wissenschaft aktuell wohl eher noch sehr verhalten ist, vgl.: Holzer, Angela; Benden, Ursula: Virtuelle Forschungsumgebungen - Quo vadunt? In: Zeitschrift für Bibliothekswesen und Bibliographie 62 (2015), S. 25-32.

${ }^{12}$ Richtlinie 2013/37/EU des Europäischen Parlaments und des Rates vom 26. Juni 2013 zur Änderung der Richtlinie 2003/98/EG über die Weiterverwendung von Informationen des öffentlichen Sektors, insbes. S. L $175 / 4$ und L $175 / 7$.
} 
${ }^{13}$ Schulze Wessel, Martin: Sammeln für die Interessen von morgen. In: Frankfurter Allgemeine Zeitung, Nr. 81, 08. April 2014.

${ }^{14}$ Vgl. Henseler, Wolfgang: Natural User Interfaces. Die Kunst, Nutzung intuitiv zu gestalten, 25. Juni 2013. http://webmagazin.de/design/user-experience/Natural-User-Interfaces-Kunst-Nutzung-intuitiv-zu-gestalten

${ }^{15}$ Thesaurus Librorum: 425 Jahre Bayerische Staatsbibliothek. Wiesbaden 1983, S. 9

${ }^{16} \mathrm{Vgl}$. den aktuellen Beitrag zur alle Jahre wiederkehrenden Diskussion um die Zukunft des Fachreferats, der schon im Titel die Abkehr vom Sammlungsgedanken forciert: Tappenbeck, Inka: Fachreferat 2020: from collections to connections. In: Bibliotheksdienst 49 (2015), S. 37-48.

${ }^{17}$ Ritter, Joachim: Hegel und die französische Revolution. In: Metaphysik und Politik. Studien zu Aristoteles und Hegel. Frankfurt/M. 2003, S. 183-233; 213 (Original 1957).

${ }^{18}$ Ritter (Anm. 17) S. 227.

19 Marquard, Odo: Zukunft braucht Herkunft. Philosophische Betrachtungen über Modernität und Menschlichkeit. In: Zukunft braucht Herkunft. Philosophische Essays. Stuttgart 2015, S. 234-246; S. 235.

${ }^{20}$ Marquardt (Anm. 19) S. 234.

${ }^{21}$ Kümmel; Strohschneider (Anm. 4) S. 125.

${ }^{22}$ Schulze Wessel (Anm. 13).

${ }^{23}$ Kümmel; Strohschneider (Anm. 4) S. 125. 